\title{
Intrinsic reinforcing properties of putatively neutral stimuli in an instrumental two-lever discrimination task
}

\author{
PHIL REED, CHRIS MITCHELL, and TRISTAN NOKES \\ University College London, London, England
}

\begin{abstract}
Four experiments examined the influence of a stimulus presented after one response in a two-lever choice task. In Experiment 1, food-deprived rats trained on a concurrent variable-interval extinction schedule responded more often on the extinction lever when such responding periodically produced a visual stimulus than when it did not. In Experiments 2 and 3, a similar signal-induced enhancement effect was found even when food was delivered randomly with respect to responding on both levers or when no food was presented. In Experiment 4, a response-contingent visual stimulus elevated responding to the lever on which it was presented, but an auditory cue suppressed responding. These findings indicate that visual stimuli may possess intrinsically reinforcing properties for rats.
\end{abstract}

Presentation of a response-contingent stimulus during a delay of reinforcement has been found to attenuate the decrement in instrumental responding that would otherwise result from that reinforcement delay (see Reed, 1992; Williams, Preston, \& deKervor, 1990). Similar signalinduced effects have been noted in studies of discrimination learning. When an animal has a choice between two alternative responses - one of which leads to reward and the other of which does not-a delay between the choice response and the delivery of reward can substantially retard the emergence of a discrimination between the responses (Grice, 1948; Lieberman, McIntosh, \& Thomas, 1979). However, if a brief, salient cue is presented following the correct response, then discrimination is improved relative to a condition in which no stimulus is presented (Reed, 1993). These effects have traditionally been ascribed to the acquisition of conditioned reinforcing properties by the stimulus (e.g., Grice, 1948). However, a number of other explanations have also been offered.

Lieberman et al. (1979; see also Lieberman, Davidson, \& Thomas, 1985), for example, suggested that presentation of a salient stimulus following a response promotes a backward scan through memory in order to identify the cause of the stimulus. The attention focused upon the response immediately preceding the stimulus supposedly produces stronger encoding of that response in memory and allows the representation of the response to survive decay across the delay period until reinforcement is delivered. The presence of the representation of the response in

The authors thank $\mathrm{C}$. Heyes for reading an earlier version of this paper and Peter Urcuioli for critical comments on several drafts of the manuscript. Requests for reprints should be addressed to P. Reed, Department of Psychology, University College London, Gower Street, London WC1 6BT, England.

-Accepted by previous editor, Vincent M. LoLordo memory when reinforcement is subsequently presented thus allows the response to be associated with the reward in a manner that would not occur if the representation decayed, as in an unsignaled control condition.

In a series of experiments designed to examine these response-contingent stimulus effects on discrimination performance by rats, Reed (1993) associated one lever in a two-lever task with variable interval (VI) 60 -sec reinforcement, but with a 5-sec delay of reward, and the other lever with an extinction (ext) schedule. For some rats, a visual stimulus was associated with responses to the ext lever. These subjects responded at a higher rate to that lever than did rats not receiving the visual stimulus. Moreover, the emergence of a discrimination between the two levers was retarded by a signal presented on the incorrect lever relative to when no signal was presented.

Reed (1993) suggested two possible mechanisms for this effect. The first was based on the possibility that the flash of light served to produce a backward scan through memory (see Lieberman et al., 1979). If this were the case, it might be expected that the stimulus would mark in memory responses to the lever associated with extinction, and that those responses would be associated with subsequent reward delivery because they would be represented strongly in memory.

Alternatively, it was suggested that the illumination of a light contingent upon a leverpress in a darkened chamber might itself act as a reinforcer. This would lead to responses being made on the lever not associated with food, since these responses would receive immediate reinforcement from the visual stimulus. This latter suggestion receives some support from studies of sensory reinforcement. Sensory reinforcement refers to the maintenance or reduction of responding through, say, visual or auditory stimulation (see Kish, 1966, for a review) and is distinct from secondary reinforcement since it is assumed that the reinforcing properties of the stimuli are not due to the associ- 
ation of the stimulus in question with a "primary" reinforcer. A number of experiments have shown that responsecontingent visual stimulation can serve to increase response rates (Kish, 1955; Watras, 1982; Winefield \& Glow, 1980; see Matsuzawa, 1981, for reviews). However, these studies have also suggested that there are optimal levels at which visual stimulation will exert a reinforcing effect, beyond which the stimulation is ineffective (Watras, 1982). Moreover, under some circumstances, light offset can be as reinforcing as light onset (Winefield \& Glow, 1980), and such effects may be modulated by the immediately preceding experience with visual stimulation (Russell \& Glow, 1974). Given these considerations, it is far from clear whether the characteristics of the experimental procedure and visual stimulus used by Reed (1993) would have led to sensory reinforcement via the visual stimulation.

Given the implications for understanding delay stimulus function, the present experiments were designed to test two accounts of the effects of presenting a stimulus following a response (i.e., marking and intrinsic reinforcement) using a visual stimulus.

\section{EXPERIMENT 1}

Experiment 1 attempted to replicate the effect reported by Reed (1993). Subjects were trained on a concurrent VI ext schedule, with reinforcement delayed by $5 \mathrm{sec}$ on the lever associated with the VI schedule. If the effect were replicated, then subjects receiving a visual stimulus during the ext component should respond more often to this lever than should subjects not receiving this stimulus.

\section{Method}

Subjects. Thirty-two male hooded Lister rats served in Experiment 1 . The subjects were 4-5 months old at the start of training, had a free-feeding body-weight range of $330-455 \mathrm{~g}$, and were maintained at $80 \%$ of this weight throughout the experiment. The subjects had previously served in an observational learning experiment in which they had pushed a joystick and experienced tone stimuli; however, they were naive with respect to leverpressing and the stimulus to be used as the delay signal. The animals were housed in groups of 4 , with water constantly available in the home cage. A 12:12-h light:dark cycle was used, with experiments conducted during the light part of the cycle.

Apparatus. Four identical operant conditioning chambers (Campden Instruments Ltd.) were used. Each chamber was housed in a light- and sound-attenuating case ventilated by a fan that provided background masking noise ( $65 \mathrm{~dB}[\mathrm{~A}])$. Each chamber had two levers, both of which were permanently inserted into the chamber. A jeweled houselight located on the ceiling of the chamber served as the stimulus in the present series of experiments. A speaker mounted on the outside of the ceiling of the chamber could deliver a $105-\mathrm{dB}(\mathrm{A})$ tone ( $40 \mathrm{~dB}$ above background). Reinforcement consisted of one 45-mg food pellet delivered to a centrally located, recessed food tray that was covered by a clear Perspex hinged flap. Apart from the visual signal, the chamber was not illuminated during the course of the experiment.

Procedure. The subjects were magazine-trained in two 30-min sessions, during which food was delivered according to a variable time (VT) 60 -sec schedule (range $=1-120 \mathrm{sec}$ ). For the first session, the magazine flap was taped open to allow easy access to the food pellets. During the second session, and for all future sessions, the flap was lowered to its standard resting position. Following magazine training, the subjects were taught to press the levers in one 30 min session, during which every response to each of the two levers was reinforced. The subjects then received two 30 -min sessions of training on a concurrent VI $30-\mathrm{sec}$ (range $=1-60 \mathrm{sec}$ ) VI $30-\mathrm{sec}$ scheduie. Following this, they were divided into two groups $(n=16)$ counterbalanced for response rate to the two levers and for the distribution of responses across levers. In all cases, the lever that supported fewer responses was chosen as the to-be-reinforced lever.

The subjects were then exposed to a concurrent VI 60-sec (range $=$ $1-120 \mathrm{sec}$ ) ext schedule. Reinforcement was delivered $5 \mathrm{sec}$ after the response that satisfied the VI 60-sec requirement. Responses during the delay period were recorded but had no programmed consequences. Responses to the other lever were never scheduled for reinforcement. One group (Group Incor), however, periodically experienced a $2-\mathrm{sec}$ response-contingent illumination of the light on the ext lever; light presentations were programmed to occur on a VI 60 $\mathrm{sec}$ schedule on the ext lever. Group Unsig received no stimulus presentations. Training continued for 1030 -min sessions.

\section{Results and Discussion}

Figure 1 displays the mean number of responses per session by each group on each lever. Inspection of the data demonstrates that responses to the lever associated with the VI 60-sec schedule remained approximately constant for both groups throughout training, although the number of responses was higher in Group Unsig than in Group Incor. In the ext component, responding declined for both groups over the course of training, but such responding was higher in Group Incor than in Group Unsig. These data were subject to a three-factor analysis of variance (ANOVA) with group (Incor vs. Unsig) as a betweensubject factor, and lever (VI vs. ext) and session as withinsubject factors. A rejection criterion of $p<.05$ was adopted for this and all subsequent analyses. There were significant main effects of lever $[F(1,30)=7.56]$ and session $[F(9,270)=6.81]$ and significant interactions between group and lever $[F(1,30)=4.43]$ and session and lever $[F(9,270)=11.52]$. No other main effects or interactions were statistically significant. To examine these data further, the simple effect of group on the last session of training for the VI 60 -sec lever and for the ext lever was analyzed. These analyses revealed that Group Unsig responded significantly more often on the VI 60 -sec lever than did Group Incor $[F(1,270)=4.88]$ but that Group Incor re-

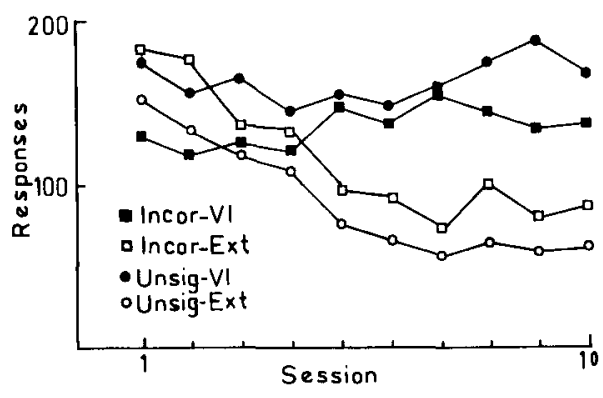

Figure 1. Mean response rates for both groups in both phases of Experiment 1. Incor $=$ stimulus presented after responses to extinction lever. Unsig $=$ no stimulus presentations. $V I=$ reinforced lever, associated with the VI 60-sec schedule. Ext $=$ nonreinforced lever, associated with extinction schedule. 
sponded more often on the ext lever than did Group Unsig $[F(1,270)=2.78]$.

The mean discrimination ratios (responses on the VI 60-sec lever divided by total responses on both levers) are displayed in Figure 2. Discrimination ratios increased slightly over the course of training for both groups and, more importantly, Group Unsig had consistently higher discrimination ratios than did Group Incor. A two-factor ANOVA (group $\times$ session) revealed significant main effects of group $[F(1,30)=5.33]$ and session $[F(9,270)=$ $11.61 \mathrm{~J}$, but no interaction between these factors.

These results replicate those reported by Reed (1993): presenting a visual stimulus on a lever not associated with food supported higher levels of responding on that lever and consistently reduced the level of discrimination between levers relative to when no signal was presented.

\section{EXPERIMENT 2}

Experiment 2 attempted to extend the generality of signal-induced effects obtained in Experiment 1. Two groups of rats were presented with two levers, but with food delivered randomly with respect to responding - that is, food was not contingent upon responding on either lever. For one group, responses on one lever were associated with the aperiodic presentation of a visual stimulus. For the other group, no stimulus was presented. If the visual stimulus serves to enhance responding via either its intrinsic reinforcing properties or a backward scan mechanism, then the response rate to the lever on which the stimulus was presented should be enhanced.

After training with these contingencies in Phase 1, the lever associated with the stimulus in the signaled group was switched. Following this Phase 2 switch, food was discontinued for both groups with the intention of discriminating between the backward scan mechanism and the intrinsic reinforcement accounts. Specifically, if the visual stimulus possesses intrinsic reinforcing properties, then responding to the lever on which the cue was presented should nonetheless be maintained at a higher level than should responses to the lever on which no stimulus was presented. However, if the stimulus were simply a marking cue, response rate to the lever associated with cue should eventually resemble that to the lever without the cue, since no

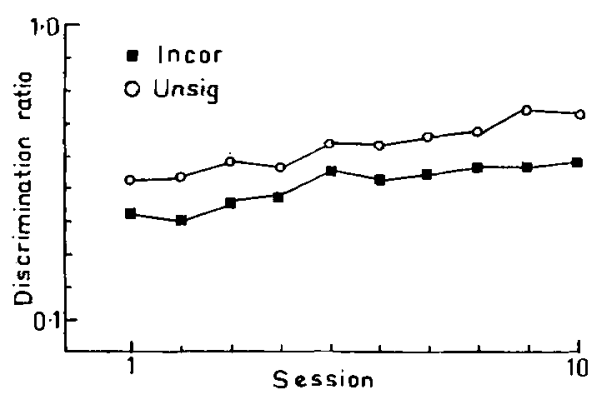

Figure 2. Mean discrimination ratios for both groups in both phases of Experiment 1. Incor $=$ stimulus presented after responses to extinction lever. Unsig $=$ no stimulus presentations. reinforcement would now be presented with which to associate a preserved representation of the response.

\section{Method}

Subjects and Apparatus. Sixteen experimentally naive male hooded Lister rats served in Experiment 2. The subjects were 3-4 months old at the start of training and had a free-feeding bodyweight range of $305-345 \mathrm{~g}$. The rats were maintained as described in Experiment 1. The apparatus was that described in Experiment 1.

Procedure. The subjects were given no pretraining. During Phase 1, food was delivered on a VT 60-sec schedule. Responses to both levers were recorded, but there was no programmed relationship between a response to either lever and the delivery of food. For Group No Stim $(n=8)$, these were the only contingencies in operation. For Group Stim ( $n=8$ ), a 2-sec flash of the overhead light was programmed to occur on a VI 60-sec schedule for one lever (Sig). For half the animals, the target lever was the right lever; for the other half, it was the left lever. This phase of training lasted for eight 30-min sessions. During Phase 2, the subjects responded on the same contingency as they had during Phase 1 except that the lever associated with the stimulus presentations in Group Stim was reversed (the lever nominally designated as the target lever in Group No Stim was similarly reversed). Phase 2 lasted for 1630 -min sessions. In Phase 3 , the subjects continued to respond on the contingencies they experienced during Phase 2 , but no food was presented to either group. This phase lasted for 1230 -min sessions.

\section{Results and Discussion}

Figure 3 displays the mean number of responses per session by each group on each lever during all phases of the experiment. The data from Phase 1 show that responding on the two levers was approximately equal in Group No Stim but that there was a markedly higher rate of response on the lever associated with the stimulus in Group Stim. A three-factor ANOVA (group $\times$ lever $\times$ session) conducted on these data revealed all main effects and interactions to be statistically significant (smallest $F=3.83$, all $p \mathrm{~s}<.05$ ). To examine these data further, the simple effect of group on the last session of Phase 1 training was analyzed for both the signaled ( $\mathrm{Sig}$ ) and the unsignaled (Unsig) levers. These tests revealed that Group Stim responded significantly more often on the signaled lever than did Group No Stim $[F(1,98)=66.61]$ but that there was no statistically significant difference between the groups on the unsignaled lever.

In Phase 2, responding was generally higher in Group Stim than in Group No Stim. Additionally, in Group Stim, responding was initially higher on the now-unsignaled (Unsig) lever than on the now-signaled (Sig) lever. This pattern of results reversed over the course of training so that, by the end of training, responding was greater on the now-signaled (Sig) lever than on the now-unsignaled (Unsig) lever. By contrast, in Group No Stim, there was little change in the level of responding on the two levers as compared with that in Phase 1. A three-factor ANOVA (group $\times$ lever $\times$ session) conducted on the Phase 2 data revealed a significant main effect of group $[F(1,14)=$ 10.67] and significant interactions between session and lever $[F(15,210)=2.66]$ and between all three factors $[F(15,210)=2.58]$. No other main effects or interactions were significant. A main effect analysis of group on the last session of training for the signaled (Sig) and the 


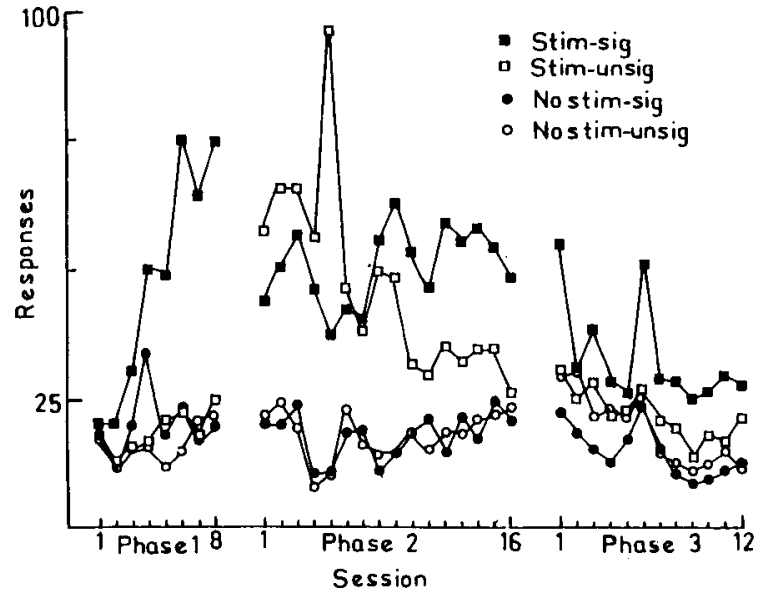

Figure 3. Mean response rates for both groups in all phases of Experiment 2. Stim = stimulus presented after responses to target lever. No stim $=$ no stimulus presentations. Sig $=$ lever associated with stimulus. Unsig $=$ lever with no stimulus presentations.

unsignaled (Unsig) levers revealed that Group Stim responded significantly more often on the signaled lever than did Group No Stim $[F(1,210)=18.53]$ but that there was no statistically significant between-group difference in responding on the unsignaled lever.

During extinction (Phase 3), responding declined on both levers in both groups. However, a difference in responding on the levers was maintained in Group Stim over the course of this phase. A three-factor ANOVA (group $X$ lever $X$ session) conducted on the Phase 3 data revealed a significant main effect of session $[F(1,14)=8.87]$ and a significant interaction between group and lever $[F(1,14)=$ 4.56]. No other main effects or interactions were significant. The simple effect of group on the last session of training was analyzed for both levers. These tests revealed that Group Stim responded significantly more often on the signaled lever than did Group No Stim $[F(1,154)=8.54]$, but that there was no statistically significant difference between the groups on the unsignaled lever.

Figure 4 shows the mean discrimination ratios (responses on the signaled lever divided by total responses on both levers) for all phases. During Phase 1, the discrimination ratio became higher for Group Stim than for Group No Stim. However, a two-factor ANOVA (group $\times$ session) conducted on these data revealed only a significant main effect of session $[F(7,98)=2.81]$. During Phase 2, the discrimination ratio remained stable in Group No Stim but increased over the course of the phase in Group Stim. A two-factor ANOVA (group $\times$ session) revealed neither main effects nor interaction to be significant. Although the analysis conducted on each phase individually proved nonsignificant, inspection of the data indicates that performance across phases altered as a result of the reversal of the signaling contingency. To confirm this observation, the discrimination ratios from the last session of Phase 1 and Phase 2 were subject to a two-factor ANOVA (lever [left vs. right] $\times$ phase), which revealed a significant in- teraction between the factors $[F(1,14)=4.15]$. During Phase 3, there was no alteration in the discrimination ratios of the two groups from their Phase 2 levels. A twofactor ANOVA (group $\times$ session) revealed a significant effect of group $[F(1,14)=4.45]$; no other effects were significant.

Taken as a whole, these data again demonstrate that when a visual stimulus is presented contingently for responding on one lever, responding is greater on that lever than when no stimulus is presented. This result mirrors that obtained in Experiment 1, although in the present experiment there was no contingent relationship between responding and the delivery of food. Furthermore, when the food was discontinued, the discrimination between the levers was maintained at Phase 2 levels in Group Stim. If the stimulus was acting solely through a backward scan mechanism, discrimination ratios would have been expected to decline over the course of extinction. The results, therefore, are more supportive of an explanation in terms of the intrinsic reinforcing properties of the stimulus. It should be noted, however, that the interpretation of the Phase 3 results is complicated by the fact that the baseline rates of response prior to extinction for the two groups, and on the two levers in Group Stim, were different.

\section{EXPERIMENT 3}

The purpose of Experiment 3 was to determine whether or not the presence of food is required to generate the signalinduced enhancement of responding. The presence of food is required to explain the signal-induced effect by the backward scan hypothesis but is not required by the intrinsic reinforcing properties account. A $2 \times 2$ factorial design was adopted, in which groups had either food delivered randomly with respect to responding or no food delivered and had either a response-contingent stimulus presented on one lever or no stimulus presented. If the backward scan hypothesis is correct, then the enhance-

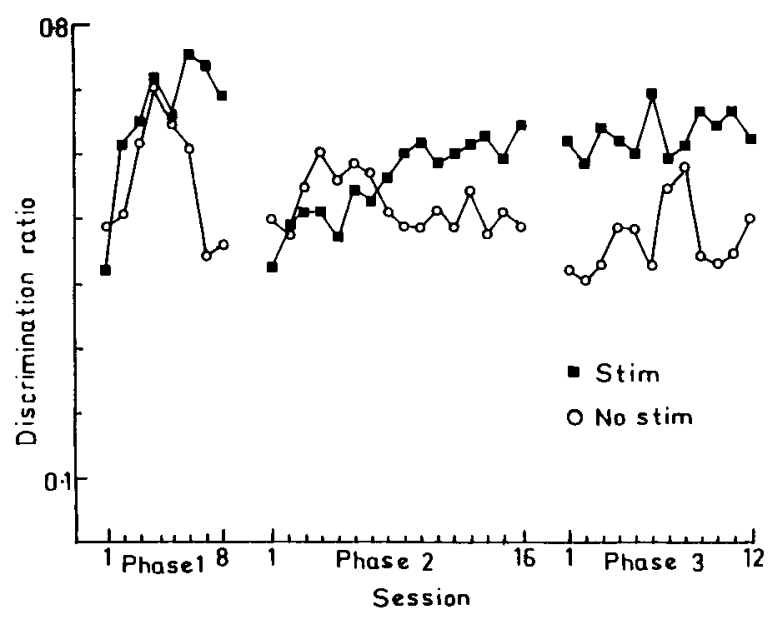

Figure 4. Mean discrimination ratios for both groups in all phases of Experiment 2. Stim = stimulus presented after responses to target lever. No stim $=$ no stimulus presentations. 
ment of responding on the signaled lever should be greater with the presence than with the absence of food. If the stimulus works primarily by its intrinsic reinforcing properties, however, then the enhancement engendered by the stimulus should be similar in these two conditions.

One consideration that suggests it might be harder to obtain signal-induced enhancement of performance in a group with food delivery is the possibility of occasional contiguous pairings of food with responses on the unsignaled lever. This might result in superstitiously conditioned responding on this lever (Skinner, 1948). Any responding maintained by immediate superstitious reinforcement on the unsignaled lever would reduce the extent to which the stimulus increased response rate on the other lever. In an attempt to curtail the possible influence of superstitious conditioning, a second phase of training was given in which the rats responded under the same conditions as described above, except that food was never delivered within $5 \mathrm{sec}$ of a response on any lever.

\section{Method}

Subjects and Apparatus. Thirty-two experimentally naive male hooded Lister rats served in Experiment 3. The subjects were 3-4 months old at the start of training and had a free-feeding bodyweight range of $320-405 \mathrm{~g}$. The rats were maintained as described in Experiment 1. The apparatus was that described in Experiment 1.

Procedure. The subjects were given no pretraining and were divided into four groups of 8 . During Phase 1, two groups (Group F-NS and Group F-S) had food delivered on a VT 60-sec schedule. Responses on both of the levers were recorded, but there was no programmed relationship between a response on either lever and the delivery of food. For the other two groups (Group NF-NS and Group NF-S), no food was delivered during training. For two of the groups (Group F-S and Group NF-S), a 2-sec flash of the overhead light was programmed to occur on a VI $60-\mathrm{sec}$ schedule contingently upon a response on one lever ( $\mathrm{Sig}$ ). For half the animals in these two groups, this lever was the right lever; for the other half, it was the left lever. For Groups F-NS and NF-NS, there were no stimulus presentations. For half the animals in these latter two groups, the right lever was taken as the target lever (Sig); for the other half, the left lever was the target lever. This initial phase of training lasted for 10 , 30-min sessions. During Phase 2, the subjects responded according to the same contingencies as in Phase 1, except that food was never presented within $5 \mathrm{sec}$ of a response. This phase also lasted for 10 30-min sessions.

\section{Results and Discussion}

Figure 5 displays the mean number of responses per session by all groups on each lever during both phases. Inspection of the data from Phase 1 reveals that responding did not increase to any great extent except on the levers associated with the stimulus (Sig) in Groups F-S and NF-S. A four-factor ANOVA (food $\times$ signal $\times$ lever $\times$ session) revealed that none of the interaction terms that involved both signal and food were significant, indicating that the signal had the same effect whether or not food was present. There were main effects of stimulus $[F(1,27)=24.58]$, food $[F(1,27)=14.44]$, lever $[F(1,27)=25.41]$, and session $[F(9,243)=6.37]$. Of the remaining interactions, that between signal and session $[F(9,243)=6.80]$, lever and session $[F(9,243)=2.71]$, signal and lever $[F(1,27)=$

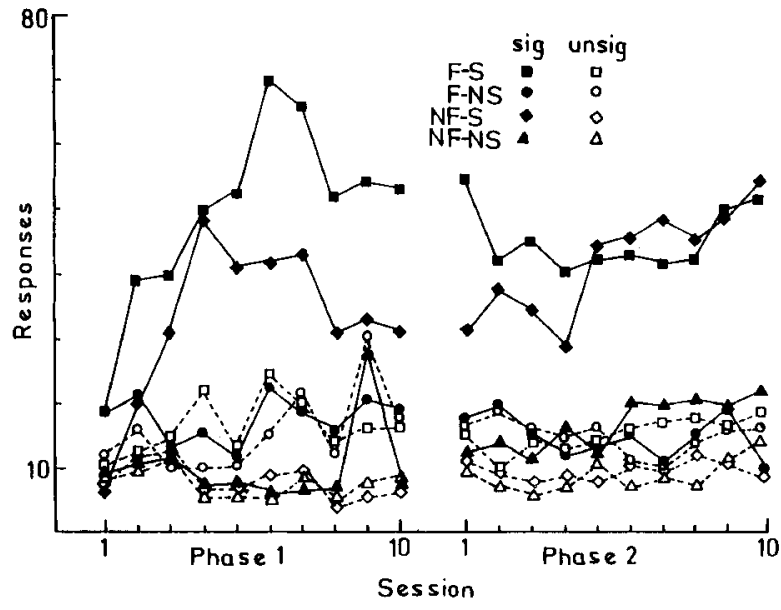

Figure 5. Mean response rates for all groups in Experiment 3. F-NS $=$ food deliveries, no stimulus presentations. $F-S=$ food deliveries, stimulus presentations. NF-NS = no food and no stimulus presentations. NF-S = no food presentations, stimulus presentations. Sig = lever associated with stimulus or designated as target lever. Unsig = lever with no stimulus presentations. Phase 2 = same contingencies except food never presented within $5 \mathrm{sec}$ of a response.

20.46], and signal, lever, and session $[F(9,243)=3.94]$ were significant.

The above pattern of results was maintained with the 5sec delay in Phase 2 . The data from Phase 2 were subjected to the same four-way ANOVA as above. As in Phase 1, there was no significant interaction involving both the food and the signal factors, indicating that the signal acted in a similar manner irrespective of the presence or absence of food. There were significant main effects of session $[F(9,243)=2.87]$, signal $[F(1,27)=26.72]$, and lever $[F(1,27)=38.59]$ and significant interactions between signal and lever $[F(1,27)=22.22]$ and between food, lever, and session $[F(9,243)=1.95]$. None of the other interactions proved significant.

The mean discrimination ratios (responses on the signaled lever divided by total responses on both levers) for both phases of the experiment are displayed in Figure 6 . These data show that discrimination ratios increased during Phase 1 in the groups with a stimulus (NF-S and F-S) but not in the groups without it. A three-factor ANOVA (food $\times$ signal $\times$ session) corroborated this observation by revealing a significant main effect of signal $[F(1,27)=$ $12.38]$ and a significant signal by session interaction $[F(9,243)=2.58]$. None of the other main effects or interactions were significant. There was little change in the discrimination ratios after the introduction of the delay contingencies in Phase 2. A three-factor ANOVA revealed that the only significant effect was the main effect of signal $[F(1,27)=12.97]$.

These data confirm that the presentation of a responsecontingent visual stimulus associated with one lever in a two-lever discrimination task will enhance rates of response on that lever. There was no evidence that food also 


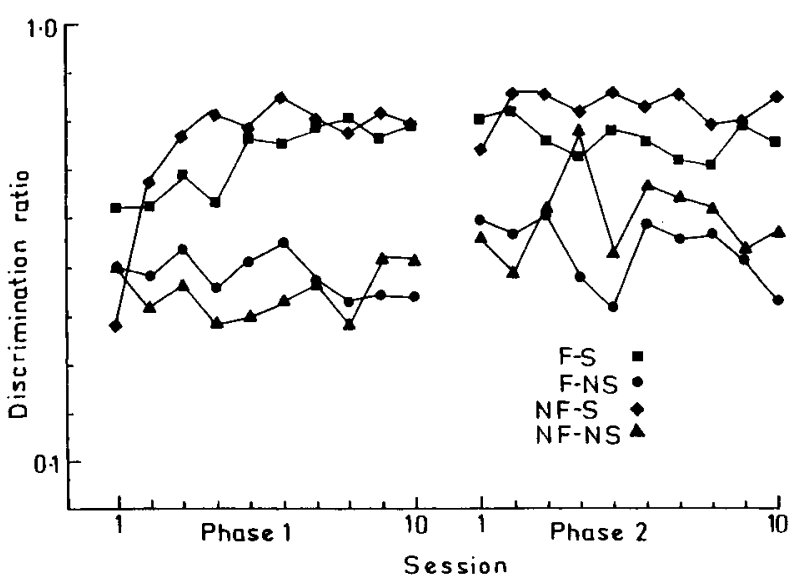

Figure 6. Mean discrimination ratios for all groups in Experiment 3. $\mathrm{F}-\mathrm{NS}=$ food deliveries, no stimulus presentations. $\mathrm{F}-\mathrm{S}=$ food deliveries, stimulus presentations on target lever. NF-NS = no food and no stimulus presentations. NF-S $=$ no food presentations, stimulus presentations on target lever. Phase 2 = same contingencies except food never presented within $5 \mathrm{sec}$ of a response.

had to be presented in order for this effect to occur. The introduction of a delay between a response and the delivery of food in Phase 2 led to a small reduction in response rate in the groups receiving food, but it did not alter the effect of visual stimulation. Thus, there was no suggestion that superstitious reinforcement of responding on the unsignaled lever masked an influence of the stimulus on responding on the signaled lever.

These findings strongly suggest that the effect of the brief stimulus is connected with its ability to reinforce responses through reinforcing properties intrinsic to the cue rather than through a backward scan mechanism.

\section{EXPERIMENT 4}

Experiment 4 had two aims: (1) to replicate the finding that the presence of a stimulus would elevate responding on the lever on which a visual stimulus was presented irrespective of whether food was also presented, and (2) to investigate whether such stimulus-induced enhancement of responding would occur when a stimulus from another modality is used. If the mere presence of a contingent relationship between a response and subsequent event is reinforcing, then enhancement in performance should also occur with stimuli from different modalities (e.g., an auditory stimulus). In contrast, if the particular properties of the stimulus are critical, then subjects receiving a visual stimulus should show the effect whereas subjects receiving an auditory stimulus should not, in view of previous research showing that auditory stimuli can have aversive properties (Azrin, 1958). Indeed, the opposite pattern of results might be obtained if the auditory stimulus is a loud tone.

\section{Method}

Subjects and Apparatus. Thirty-two experimentally naive male hooded Lister rats served in Experiment 4. The subjects were 4-5 months old at the start of training and had a free-feeding bodyweight range of $380-465 \mathrm{~g}$. The rats were maintained as described in Experiment 1. The apparatus was that described in Experiment 1.

Procedure. The subjects were given no pretraining and were divided into four groups of 8. For two groups (Group Food-Light and Group Food-Tone), food was delivered on a VT 60 -sec schedule Responses on both levers were recorded, but there was no programmed relationship between a response on either lever and the delivery of food. For the other two groups (Group No Food-Light and Group No Food-Tone), no food was delivered during training. Groups Food-Light and No Food-Light had a 2-sec flash of the overhead light programmed to occur on a VI 60-sec schedule for one lever (Sig), which was counterbalanced across subjects in each group. For Groups Food-Tone and No Food-Tone, the stimulus was a $2-\mathrm{sec}$ presentation of a $105-\mathrm{dB}(\mathrm{A})$ tone ( $40 \mathrm{~dB}$ above background) scheduled in the same way as the light stimulus. Training lasted for eight 25 -min sessions.

\section{Results and Discussion}

Figure 7 displays the mean number of responses per session by all groups on each lever. Groups Food-Light and No Food-Light responded more often on the lever associated with the light stimulus than they did on the other lever. By contrast, in the two tone groups, responding was, if anything, more pronounced on the lever lacking the stimulus (Unsig). A four-factor ANOVA (food [presence vs. absence] $\times$ signal [presence vs. absence] $\times$ lever [signaled vs. unsignaled] $\times$ session) corroborated this description by revealing a significant interaction between modality, lever, and session $[F(7,196)=11.54]$. To further analyze this interaction, the simple effect of signaling was analyzed separately for each group on the last session. Responding on the target lever in both of the groups with a visual stimulus was significantly greater than that on the nontarget lever [smallest $F(1,196)=37.52$ ]. The differences between the target and nontarget levers in the groups with an auditory stimulus were not significant.

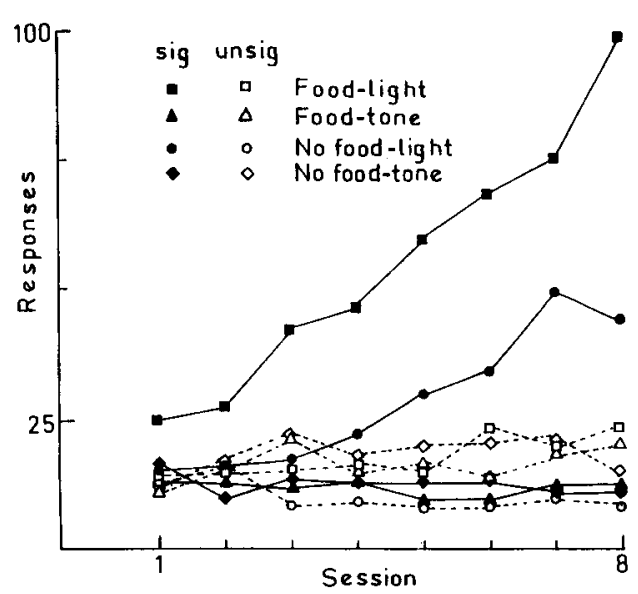

Figure 7. Mean response rates for all groups in Experiment 4. Food-tone $=$ food presented, auditory stimulus presentations. Food-light $=$ food presented, visual stimulus presentations. No food-tone $=$ no food presentations, auditory stimulus presentations. No food-light $=$ no food presentations, visual stimulus presentations. Sig = lever associated with stimulus or designated as target. Unsig = lever with no stimulus presentations. 
The mean discrimination ratios (responses on the signaled lever divided by total responses on both levers) are displayed in Figure 8. These data show that discrimination ratios increased in the groups with a visual stimulus but declined over the course of training for the groups with a tone stimulus. A three-factor ANOVA (food $\times$ signal $\times$ session) corroborated this observation by revealing a significant main effect of modality $[F(1,28)=26.82]$ and a significant modality $\times$ session interaction $[F(7,196)=$ 7.34]. No other main effects or interactions were significant. A $t$ test conducted on the combined data from the groups with a light stimulus revealed that their discrimination ratio on the last session of training was significantly greater than $.50[t(15)=13.62]$. A $t$ test conducted on the combined data from the tone groups revealed their discrimination ratio to be significantly lower than .50 $[t(15)=1.76]$.

These data replicate the finding that the presence of a visual stimulus associated with one lever in a two-lever discrimination task will enhance rates of response on that lever and will do so irrespective of whether or not food is also presented during the session. However, this effect did not occur with an auditory stimulus. The contrasting results obtained when the auditory cue was used support the claim that intrinsic reinforcing properties of the stimulus play a role in modulating behavior in such experiments.

\section{GENERAL DISCUSSION}

The present experiments replicated the previously reported result that presentation of a visual stimulus on the incorrect lever in a two-lever discrimination task enhances the response rate to that lever and impairs discrimination performance (Experiment 1). Furthermore, it was demonstrated that the enhancement effect occurred irrespective of whether or not food was delivered (Experiments 2, 3, and 4). Together, these data imply that the visual stimulus operated to enhance performance via its intrinsic rein-

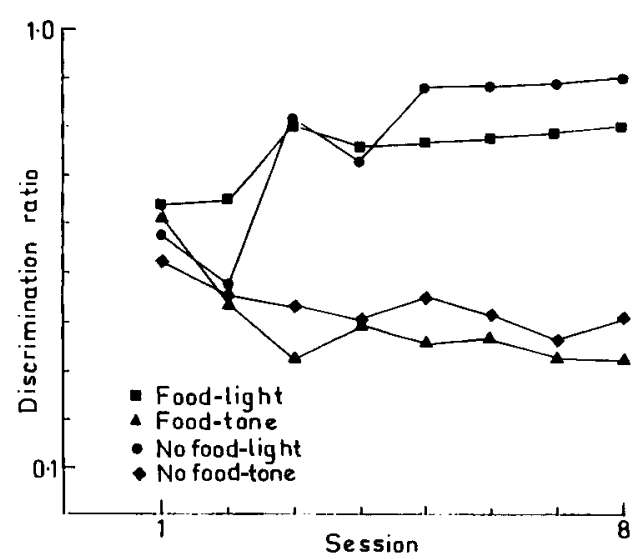

Figure 8. Mean discrimination ratios for all groups in Experiment 4. Food-tone $=$ food presentations, auditory stimulus presentations. Food-light $=$ food presentations, visual stimulus presentations. No food-tone $=$ no food, auditory stimulus presentations. No food-light $=$ no food, visual stimulus presentations. forcing properties rather than through a backward scan mechanism. This suggestion received additional support in Experiment 4, which showed that a visual stimulus promoted responding on the lever on which it was presented whereas an auditory stimulus did not.

It should be noted that some of the present results might be explained in terms of acquired secondary reinforcing properties. For example, it is possible that the stimulus sometimes occurred coincidently with the delivery of food. If such temporally contiguous pairings happened often enough, the stimulus might have acquired reinforcing properties that would then have maintained responding on the lever on which it was presented. However, this explanation cannot accommodate all the results, especially those obtained in Experiment 4. In the latter experiment, the effect of the stimulus on responding was equivalent in groups that received food presentation and those that did not. Moreover, in Experiment 4, the groups with the auditory stimulus did not show an enhancement effect even when food was presented. Thus, secondary reinforcement does not provide a comprehensive account of all the present findings.

Apart from corroborating the suggestion that such cues possess intrinsic reinforcing or punishing properties (see Reed, 1993), these results have implications for studies investigating the effect of presenting putatively neutral stimuli in choice tasks. First, they imply that in order for a stimulus to serve as a marking cue, an explicit contingency between the response and reinforcement should exist. In previous demonstrations of the marking effect, when such a contingent relationship between response and reinforcer existed, a signal-induced enhancement of performance has been noted relative to appropriate control conditions. No evidence for a signal-induced enhancement of performance was obtained in the present experiments. A major difference between the present procedures and those previously used is the lack of explicit contingency between response and reinforcement. Previous experiments on signalinduced effects have appeared to imply that this effect might have some parallels with superstitious conditioning: as long as a marking cue has occurred contingent upon a response, when a reinforcer is delivered at some later time, the representation of the response becomes associated with the reinforcer irrespective of the actual contingency between response and reward (see Lieberman \& Thomas, 1986). The failure to note any evidence for such an effect in the present experiments suggests that the marking effect will only occur when there is a contingent relationship between response and reinforcement.

Second, it is clear from the present experiments that putatively neutral stimuli, especially the intense stimuli known to be required for marking to occur (Thomas, Robertson, \& Lieberman, 1990), may influence performance irrespective of any other function such stimuli may serve. The present results support views regarding the function of delay stimuli that stress that different types of cue (e.g., those from different modalities, or those that are localized or diffuse) will have different effects on performance (Iversen, 1981; Reed, 1992; Reed, Schachtman, \& Hall, 1988; 
Williams et al., 1990). In studies investigating the mechanisms underlying marking in which only one of the events is followed by a marking cue, the effects of the stimulus due to its intrinsic reinforcing properties could be confounded with its effects as a marking stimulus. Any thorough understanding of the determinants of the "marking effect" will require that these confounding variables be disentangled experimentally.

A final implication of these findings concerns differential outcome effects using putatively neutral stimuli. Fedorchak and Bolles (1986), for example, demonstrated that, in a conditional discrimination procedure with rats, a visual cue presented after the correct response enhanced discrimination performance. By contrast, when the visual cue was presented after the incorrect response, the cue had no effect. Given the present findings, it may be that the intrinsic reinforcing properties of the visual stimulus were responsible for these findings. For example, the enhanced discrimination might have been the result of the presentation of two reinforcers following correct responses (the visual cue and the "biologically relevant" stimulus, water or sucrose). The lack of a differential outcome effect in the incorrect-response condition might reflect reinforcement of incorrect responses by the presentation of the visual cue. In other words, both correct and incorrect responses received some reinforcement (albeit from different sources), so the conditional discrimination would be relatively difficult to learn. Of course, the fact that discrimination was not retarded in this group, relative to a group receiving no visual stimulus, implies that a differential outcome effect may also have been operating, although it may have been diminished by the reinforcing action of the visual cue.

\section{REFERENCES}

Azrin, N. H. (1958). Some effects of noise on human behavior. Journal of the Experimental Analysis of Behavior, 1, 183-200.

FedorchaK, P. M., \& Bolles, R. C. (1986). Differential outcome effect using a biologically neutral outcome difference. Journal of Experimental Psychology: Animal Behavior Processes, 12, 125-130.

GricE, G. R. (1948). The relation of secondary reinforcement to delayed reward in visual discrimination learning. Journal of Experimental Psychology, 38, 1-6.
IVERSEN, I. H. (1981). Response interaction with signalled delay of reinforcement. Behavior Analysis Letters, 1, 3-9.

KISH, G. B. (1955). Learning when the onset of illumination is used as reinforcing stimulus. Journal of Comparative \& Physiological Psychology, 48, 261-264.

KISH, G. B. (1966). Studies of sensory reinforcement. In W. K. Honig (Ed.), Operant behavior: Areas of research and application (pp. 109. 159). New York: Appleton-Century-Crofts.

Lieberman, D. A., Davidson, F. H., \& Thomas, G. V. (1985). Marking in pigeons: The role of memory in delayed reinforcement. Journal of Experimental Psychology: Animal Behavior Processes, 11, 611-624.

Lieberman, D. A., MCINTOSH, D. C., \& Thomas, G. V. (1979). Learning when reward is delayed: A marking hypothesis. Journal of Experimental Psychology: Animal Behavior Processes, 5, 224-242.

Lieberman, D. A., \& Thomas, G. V. (1986). Marking, memory, and superstition in the pigeon. Quarterly Journal of Experimental Psychology, 38B, 449-459.

MatsuzaWA, T. (1981). Sensory reinforcement: The variety of reinforcers. Japanese Psychological Review, 24, 220-251.

REED, P. (1992). Signalled delay of reward: Overshadowing versus signtracking explanations. Learning \& Motivation, 23, 27-42.

REED, P. (1993). Differential outcome effect does not control performance in a two-choice discrimination task with visual stimuli. Learning \& Motivation, 24, 101-118.

Reed, P., Schachtman, T. R., \& Hall, G. (1988). Potentiation of responding on a VR schedule by a stimulus correlated with reinforcement: Effects of diffuse and localized signals. Animal Learning \& Behavior, 16, 75-82.

Russell, A., \& GLow, P. H. (1974). Some effects of short-term immediate prior exposure to light change on responding for light change. Animal Learning \& Behavior, 2, 262-266.

SKINNER, B. F. (1948). "Superstition" in the pigeon. Journal of Experimental Psychology, 38, 168-172.

Thomas, G. V., Robertson, D., \& Lieberman, D. A. (1990). The effects of relative intensity of cue and marker on marked trace conditioning in pigeons. Quarterly Journal of Experimental Psychology, 42B, 267-287.

WATRAS, S. (1982). Need for light stimulation and threshold of selfstimulation response in the white rat. Polish Psychological Bulletin, 13, 267-271.

Williams, B. A., Preston, R. A., \& deKervor, D. E. (1990). Blocking of the response-reinforcer association: Additional evidence. Learning \& Motivation, 21, 379-398.

WINEFIELD, A. H., \& GLOW, P. H. (1980). Active versus passive control in sensory contingent bar pressing in rats. Journal of General Psychology, 103, 27-40.

(Manuscript received February 9, 1994; revision accepted for publication September 14, 1994.) 